\title{
Signs of the Ising Model Ursell Functions
}

\author{
S. B. Shlosman
}

Institute for Problems of Information Transmission, Academy of Sciences, 101447, Ermolovoy, 19, Moscow, USSR

\begin{abstract}
It is proven that the Ursell functions $U_{2 k}$ of the Ising model have the conjectured signs: $(-1)^{k+1} U_{2 k} \geqq 0$. The proof is based on Aizenman's random current representation and combinatorics.
\end{abstract}

\section{Introduction}

The Ursell function $U_{k}\left(\sigma_{1}, \ldots, \sigma_{k}\right)$ of a family of $k$ random variables $\sigma_{1}, \ldots, \sigma_{k}$ is defined by means of a generating function:

$$
U_{k}\left(\sigma_{1}, \ldots, \sigma_{k}\right)=\left.\frac{\partial^{k}}{\partial h_{1} \ldots \partial h_{k}} \ln \left\langle\exp \sum_{i=1}^{k} h_{i} \sigma_{i}\right\rangle\right|_{h \equiv 0} .
$$

Here $\langle>$ stands for expectation. Another way to define them is by the formula

$$
U_{k}\left(\sigma_{1}, \ldots, \sigma_{k}\right)=\sum_{\mathscr{P}}(-1)^{|\mathscr{P}|-1}(|\mathscr{P}|-1) ! \sum_{P \in \mathscr{P}}\left\langle\prod_{p \in P} \sigma_{p}\right\rangle
$$

where the summation is over all partitions $\mathscr{P}$ of the set $I=\{1, \ldots, k\}$, $\mathscr{P}=\left\{P_{1}, \ldots, P_{r}\right\},|\mathscr{P}|=r, \bigcup_{i=1}^{r} P_{i}=I, P_{i} \cap P_{j}=\phi, i \neq j$. The formula (2) follows from (1) by a straightforward calculation.

In this paper we study the Ursell functions of the general Ising ferromagnet with pair interaction. We have a collection of random variables $\sigma_{1}, \ldots, \sigma_{N}, \sigma_{i}= \pm 1$, whose joint distribution is given by the probabilities

$$
P_{N}(\sigma)=Z^{-1} \exp \left\{\sum_{s, t=1, s \neq t}^{N} J_{s t} \sigma_{s} \sigma_{t}\right\},
$$

where the partition function

$$
Z=\sum_{\sigma} \exp \left\{\sum_{s, t=1, s \neq t}^{N} J_{s t} \sigma_{s} \sigma_{t}\right\},
$$

and $J_{s t} \geqq 0$ for all $s, t=1, \ldots, N$. 
It has been conjectured by many people including Jaffe, Newman [1], Feldman [2], that the Ursell functions $U_{2 k}$ of a finite Ising ferromagnet with two-body interactions obey the inequality:

$$
(-1)^{k+1} U_{2 k}\left(\sigma_{s_{1}}, \ldots, \sigma_{s_{2 k}}\right) \geqq 0 .
$$

For $k=1$ this is a special case of the second Griffiths inequality [3]. For $k=2$ it follows from the GHS inequality [4]; the corresponding inequality is called Lebowitz inequality, [5]. Cartier [6], Percus [7], and Sylvester [8] have proved (5) independently for $k=3$.

The Lebowitz inequality $U_{4} \leqq 0$ turned out to be very popular recently, when the questions of triviality of certain scaling limits and quantum field theories were considered $[9,10]$. For this, the representation through random currents was used in [9]; in particular, a very nice representation for the $U_{4}$ function was obtained [see Eq. (11) below], which is manifestly nonpositive.

This representation was the starting point for the present work. We shall show that similar representations hold for higher order Ursell functions. In particular, it enables us to prove the following:

Theorem 1. For the Ising ferromagnet with two-body interaction the inequality (5) holds.

\section{Random Currents Representation}

The starting point of Aizenman's representation is the following variant of the high-temperature expansion. Denoting by $b=\{s, t\}$ a bond, joining a pair of interacting sites, with $J_{b}=J_{s t}$, one has for the partition function

$$
Z=\sum_{\sigma} \prod_{b} \exp \left\{J_{b} \sigma_{s} \sigma_{t}\right\}
$$

Expanding each exponent, and performing the summation over $\sigma$, one arrives at the following representation:

$$
Z=\sum_{n: \partial n=\emptyset} w(n) .
$$

Here $n$ varies over the set of functions which assign an integer $n_{b}$ to each bond $b=\{s, t\}$ of the lattice, $s, t=1, \ldots, N, s \neq t$, the weight

$$
w(n)=\prod_{b}\left(J_{b}\right)^{n_{b}} /\left(n_{b}\right) !
$$

and the set $\partial n$ is defined as

$$
\partial n=\left\{s: \sum_{b: s \in b} n_{b}=\text { odd }\right\}
$$

(so that $\partial n$ is mod2-boundary). In the same way, for the correlation function

$$
\left\langle\sigma_{s} \ldots \sigma_{t}\right\rangle=\sum_{\sigma} \sigma_{s} \ldots \sigma_{t} P_{N}(\sigma)
$$

one has

$$
\left\langle\sigma_{s} \ldots \sigma_{t}\right\rangle=\sum_{\substack{n: \\ \partial n=\{s, \ldots, t\}}} w(n) / \sum_{n: \partial n=\emptyset} w(n) .
$$


For a given current $m=\left\{m_{b}\right\}$, let us decompose the set of sites into clusters, which are connected by bonds with nonvanishing $m_{b}$. Let $\mathscr{C}_{m}(s)$ be the connected cluster, containing the site $s$.

Let now $A_{1}, \ldots, A_{k} \subset\{1, \ldots, N\}$ be even subsets. Consider the ensemble $\left\{n^{1}, \ldots, n^{k} / A_{1}, \ldots, A_{k}\right\}$ of the currents, such that $\partial n^{i}=A_{i}$, and the statistical weight of a given sequence $n^{1}, \ldots, n^{k}$ is equal to $w\left(n^{1}\right), \ldots, w\left(n^{k}\right)$, so that

$$
\operatorname{Pr}\left\{n^{1}, \ldots, n^{k} / A_{1}, \ldots, A_{k}\right\}=\frac{w\left(n^{1}\right) \ldots w\left(n^{k}\right)}{\sum_{\partial n^{1}=A_{1}} w\left(n^{1}\right) \ldots \sum_{\partial n^{k=A_{k}}} w\left(n^{k}\right)} .
$$

In other words, this is the ensemble of non-interacting currents.

The following identity was obtained in [9]:

$$
\begin{aligned}
& U_{4}\left(\sigma_{s_{1}}, \sigma_{s_{2}}, \sigma_{s_{3}}, \sigma_{s_{4}}\right)=-2\left\langle\sigma_{s_{1}}, \sigma_{s_{2}}, \sigma_{s_{3}}, \sigma_{s_{4}}\right\rangle \\
& \quad \cdot \operatorname{Pr}\left\{s_{2}, s_{3}, s_{4} \in \mathscr{C}_{n^{1}+n^{2}}\left(s_{1}\right) /\left\{s_{1}, s_{2}, s_{3}, s_{4}\right\}, \emptyset\right\},
\end{aligned}
$$

where the probability of the following event is considered: the connected cluster $\mathscr{C}_{n^{1}+n^{2}}\left(s_{1}\right)$ of the current $n^{1}+n^{2}$, which contains the site $s_{1}$, contain also three other sites $s_{2}, s_{3}, s_{4}$. Here $\left(n^{1}+n^{2}\right)_{b}$ is $n_{b}^{1}+n_{b}^{2}$. Note that, in general, the cluster $\mathscr{C}_{n^{1}+n^{2}}\left(s_{1}\right)$ contains one of the three sites $s_{2}, s_{3}, s_{4}$, but does not necessarily contain the rest of them.

Going on to general values of $k$, we can obtain a similar representation, using the formulas (2), (6), (8).

Namely, one has

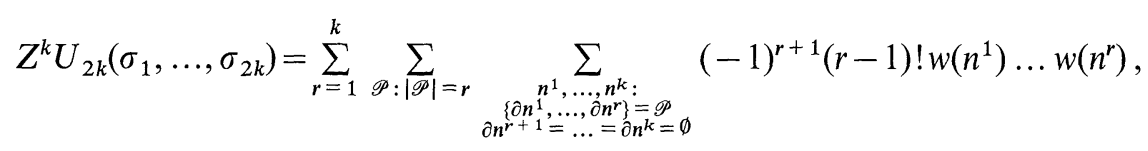

where the first summation goes over all partitions $\mathscr{P}$ of the set $\{1, \ldots, 2 k\}$ into $r=|\mathscr{P}|$ even subsets, $\mathscr{P}=\left\{P_{1}, \ldots, P_{r}\right\}$, whereas the inner summation runs over all collections of $k$ currents $n^{1}, \ldots, n^{k}$, such that $\partial n^{i}=\left\{s_{j_{1}}, \ldots, s_{j_{2}}\right\}$, where $\left\{j_{1}, \ldots, j_{i}\right\}=P_{i}$, provided $i \leqq r, \partial n^{i}=\emptyset$ otherwise. Changing the variables according to the formula $\left(n^{1}, n^{2}, \ldots, n^{k}\right) \rightarrow\left(m=n^{1}+\ldots+n^{k}, n^{2}, \ldots, n^{k}\right)$, one arrives at the following identity:

$$
\begin{aligned}
& Z^{k} U_{2 k}\left(\sigma_{1}, \ldots, \sigma_{2 k}\right)=\sum_{\partial m=\{1, \ldots, 2 k\}} w(m) \sum_{r=1}^{k} \sum_{\mathscr{P}:|\mathscr{P}|=r}(-1)^{r-1}(r-1) !
\end{aligned}
$$

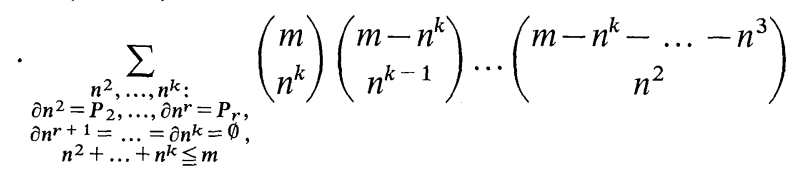

$$
\begin{aligned}
& \equiv \sum_{\substack{m: \\
\partial m=\{1, \ldots, 2 k\}}} w(m) \sum_{\mathscr{P}}(-1)^{|\mathscr{P}|-1}(|\mathscr{P P}|-1) ! R(m, \mathscr{P}) \\
& \equiv \sum_{\substack{m: \\
\partial m=\{1, \ldots, 2 k\}}} w(m) R(m) .
\end{aligned}
$$


Here $\left(\begin{array}{c}m \\ n\end{array}\right)$ is a shorthand for $\prod_{b}\left(\begin{array}{c}m_{b} \\ n_{b}\end{array}\right)$, where $m_{b} \geqq n_{b} \geqq 0$, while the two last lines are the definitions of $R(m, \mathscr{P}), R(m)$. It follows easily from the above definitions, that

$$
R(m)=\left.\frac{\partial^{m_{b_{1}}+m_{b_{2}}+\ldots}}{\partial J_{b_{1}}^{m_{b_{1}}} \partial J_{b_{2}}^{m_{b_{2}} \ldots}} Z^{k} U_{2 k}\left(\sigma_{1}, \ldots, \sigma_{2 k}\right)\right|_{J \equiv 0} .
$$

What we are going to show is that

$$
(-1)^{k+1} R(m) \geqq 0 \text { for all } m \geqq 0 .
$$

Clearly, it implies (5), because of (12).

In fact, the inequality (14) was conjectured already in [8], where it was checked for $k=1,2,3$. The technique of [8] does not permit one, however, to prove (14) for other values of $k$. What enables us to prove (14) is the precise combinatorial meaning of the quantities $R(m)$, which is given by (12) and which we are going to exploit in the next section. We close this section by the observation that it enough to prove (5) only for the case when all the sites $s_{1}, \ldots, s_{2 k}$ are mutually distinct, since otherwise one can exploit the reduction formula, expressing the given Ursell function with some coincident arguments through lower order Ursell functions, see e.g. [8].

\section{The Combinatoric Theorem}

In this section the main result of this paper - the bound (14) - is proven. Since this result is purely combinatoric, we shall state it once more in combinatoric terms and in closed form.

Let $G$ be any graph, i.e. a finite set $V(G)$ of sites and a finite set $B(G)$ of bonds are given, together with a map $\pi: B(G) \rightarrow V(G) \times V(G) / \mathbb{Z}_{2}$, where the action of the nontrivial element $g \in \mathbb{Z}_{2}$ on $V(G) \times V(G)$ is given by $g\left(v_{1}, v_{2}\right)=\left(v_{2}, v_{1}\right)$. In other words, for any bond $b \in B(G), \pi(b)$ is the (unordered) pair of its endpoints. The particular cases when $\pi(b)=(v, v)$ for some $v \in V(G)$, or when $\#\left(\pi^{-1}\left(v_{1}, v_{2}\right)\right)>1$ are not excluded. The map $\pi$ is called the incidence map.

For $v \in V(G)$ let $S(v)=\{b \in B(G): v \in \pi(b)\}$. The boundary $\partial G \subset V(G)$ is formed by all sites $v \in V(G)$, such that the number \# $(S(v))$ is odd. It is immediately seen that \# $\partial G$ is even.

A subgraph $\Gamma \subset G$ is a subset $B(\Gamma) \subset B(G)$, and $V(\Gamma)$ is by definition the same as $V(G)$.

By a partition $\mathscr{T}$ of the graph $G$ with $\#(\partial G)=2 k$ we mean any sequence $\Gamma_{1}, \ldots, \Gamma_{k}$ of $k$ subgraphs of $G$, such that

1) the sequence $B\left(\Gamma_{1}\right), \ldots, B\left(\Gamma_{k}\right)$ forms a (disjoint) partition of $B(G)$,

2) for all $i=1, \ldots, k \partial \Gamma_{i} \subset \partial G$,

3) the sequence $\partial \Gamma_{1}, \ldots, \partial \Gamma_{k}$ forms a (disjoint) partition of $V(G)$, i.e. each site $v \in \partial G$ belongs to the boundary of exactly one subgraph.

Let $n(\mathscr{T})$ be the number of the subgraphs in the partition $\mathscr{T}$ with nonempty boundaries. Clearly, $1 \leqq n(\mathscr{T}) \leqq k$. We shall suppose that the numbering of subgraphs $\Gamma_{i}$ is so adjusted that $\partial \Gamma_{i} \neq \emptyset$ whenever $i \leqq n(\mathscr{T})$.

Two partitions $\mathscr{T}^{\prime}=\left(\Gamma_{1}^{\prime}, \ldots, \Gamma_{k}^{\prime}\right)$ and $\mathscr{T}^{\prime \prime}=\left(\Gamma_{1}^{\prime \prime}, \ldots, \Gamma_{k}^{\prime \prime}\right)$ are called distinct, if

A) two partitions $\left(\partial \Gamma_{1}^{\prime}, \ldots, \partial \Gamma_{n\left(\mathscr{T}^{\prime}\right)}^{\prime}\right)$ and $\left(\partial \Gamma_{1}^{\prime \prime}, \ldots, \partial \Gamma_{n\left(\mathscr{T}^{\prime \prime}\right)}^{\prime \prime}\right)$ are distinct as unordered partitions [i.e. for some $i \leqq n\left(\mathscr{T}^{\prime}\right), \partial \Gamma_{i}^{\prime} \neq \partial \Gamma_{j}^{\prime \prime}$ for all $j$ ]; otherwise if 
B) for some $i, j, 1 \leqq i, j \leqq n\left(\mathscr{T}^{\prime}\right)=n\left(\mathscr{T}^{\prime \prime}\right), \partial \Gamma_{i}^{\prime}=\partial \Gamma_{j}^{\prime \prime} \neq \emptyset$, but $\Gamma_{i}^{\prime} \neq \Gamma_{j}^{\prime \prime}$; otherwise if

C) for some $j>n\left(\mathscr{T}^{\prime}\right)=n\left(\mathscr{T}^{\prime \prime}\right), \Gamma_{j}^{\prime} \neq \Gamma_{j}^{\prime \prime}$ (which means that the numbering of the subgraphs with empty boundary is essential).

Let us define now the quantity $R(G)$ :

$$
R(G)=\sum_{\mathscr{T}}(-1)^{(n(\mathscr{T})-1)}(n(\mathscr{T})-1) !,
$$

where the sum goes over all distinct partitions of the graph $G$. It is straightforward to see that our $R(G)$ is the same as $R(m)$ of the preceding section.

Theorem 2. For all finite graphs $G$ with $\# \partial G=k$,

$$
(-1)^{k+1} R(G) \geqq 0 \text {. }
$$

Moreover, if $G$ is a disjoint union of two graphs $G_{1}, G_{2}$, with $\partial G_{1} \neq \emptyset \neq \partial G_{2}$, then $R(G) \equiv 0$.

Proof. The proof goes on by induction on the number of bonds, \# $(B(G))$.

Suppose first that there is a loop in the graph $G$, i.e. a bond $b \in B(G)$, such that $\pi(b)=(v, v)$ with $v \in V(G)$. In that case we can pass to the graph $G^{\prime}=G \backslash b$, and because to each partition of $G^{\prime}$ there corresponds exactly $k$ partitions of $G$ namely, the loop $b$ can be attached to any of $k$ subgraphs $\Gamma_{1}, \ldots, \Gamma_{k}$-one has: $R(G)$ $=k R\left(G^{\prime}\right)$. (Note that in the above argument, the condition $\mathrm{C}$, dealing with the distinctness of two partitions, is of importance.)

Consider now the case when $G$ contains a site $v$, which is incident to exactly two bonds $b_{1}, b_{2} \in B(G)$. Then, for any partition $\mathscr{T}$ of $G$, these bonds necessarily belong to the same subgraph $\Gamma$ of the partition - because if $b_{1} \in \Gamma_{1}$ and $b_{2} \in \Gamma_{2} \neq \Gamma_{1}$, then $v \in \partial \Gamma_{1}, v \in \partial \Gamma_{2}$ while $v \notin \partial G$, and so the definition of $\mathscr{T}$ to be a partition is violated. So, in this case, if $\pi\left(b_{1}\right)=\left(v, v_{1}\right), \pi\left(b_{2}\right)=\left(v, v_{2}\right)$, then one can pass to the graph $G^{\prime \prime}$ with

$$
V\left(G^{\prime \prime}\right)=V(G) \backslash v, \quad B\left(G^{\prime \prime}\right)=\left[B(G) \backslash\left(b_{1} \cup b_{2}\right)\right] \cup b_{12},
$$

and with $\pi\left(b_{12}\right)=\left(v_{1}, v_{2}\right)$, and $\pi$ unchanged for other bonds.

From the above discussion,

$$
R(G)=R\left(G^{\prime \prime}\right) .
$$

But $\# B(G)>\# B\left(G^{\prime}\right)$, \#B(G'), so that the induction step is made.

It remains to consider the case when for all sites $v$ the number of incident bonds, $\# S(v)$, is different from 2. Suppose $S(v) \geqq 3$ for some $v$, and $b_{1}, b_{2} \in S(v), \pi\left(b_{1}\right)$ $=\left(v_{1}, v\right), \pi\left(b_{2}\right)=\left(v_{2}, v\right)$. Let us split the quantity $R(G)$ :

$$
R(G)=R\left(G ;\left[b_{1}, b_{2}\right]\right)+R\left(G ;\left\{b_{1}, b_{2}\right\}\right),
$$

where the first summand corresponds to all partitions with bonds $b_{1}, b_{2}$ belonging to the same subgraph, and the second to the remaining ones. The first term can be treated as above: one can pass to a new graph $G^{\prime \prime \prime}$ with $V\left(G^{\prime \prime \prime}\right)=V(G) \cup \tilde{v}, B\left(G^{\prime \prime \prime}\right)$ $=B(G)$, and with

$$
\pi\left(b_{1}\right)=\left(v_{1}, \tilde{v}\right), \quad \pi\left(b_{2}\right)=\left(v_{2}, \tilde{v}\right),
$$

$\pi$ unchanged for other bonds. It is evident that $R\left(G ;\left[b_{1}, b_{2}\right]\right)=R\left(G^{\prime \prime \prime}\right)$; at the same time $\# S(\tilde{v})=2$, and so the number of bonds of the graph $G$ can be reduced as 


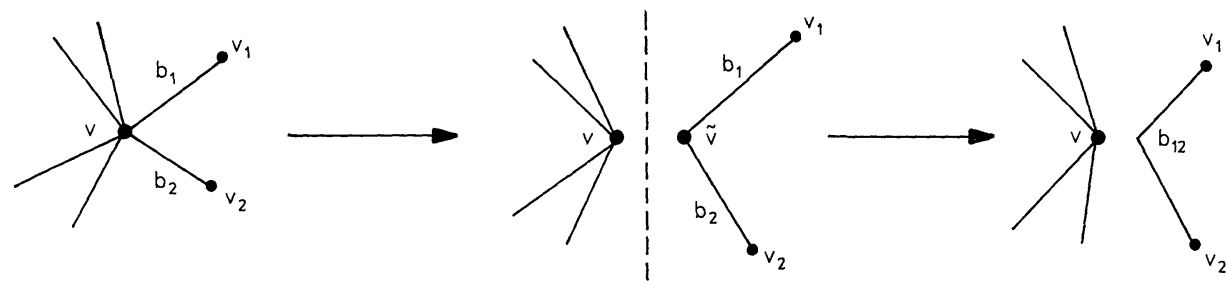

Fig. 1. The decomposition of the graph $G$ for the case when two bonds $b_{1}, b_{2}$ share the same element of a partition

above $\left(G^{\prime \prime \prime} \rightarrow\left(G^{\prime \prime \prime}\right)^{\prime \prime}\right)$, and so the first summand in (17) has the necessary sign by induction (see Fig. 1).

As for the second term, nothing can be said about its sign at this step. So, as is usual in induction proof, one has to prove a stronger result: namely, we are bound to show the following:

Theorem 3. Let $G$ be any finite graph, \# $\partial G=2 k$, and an arbitrary sequence

$$
\left(b_{i_{1}}, b_{j}\right), \ldots,\left(b_{i_{n}}, b_{j_{n}}\right)
$$

of pairs of bonds is given. Then

$$
(-1)^{k+1} R\left(G ;\left\{b_{i_{1}}, b_{j_{1}}\right\}, \ldots,\left\{b_{i_{n}}, b_{j_{n}}\right\}\right) \geqq 0,
$$

where the last quantity is defined by the formula (15) with the summation range restricted to those partitions, where for any $p=1, \ldots, n$, the bonds $b_{i_{p}}, b_{j_{p}}$ belong to different subgraphs.

The proof of Theorem 3 will be at the same time the continuation of that of Theorem 2. Again we proceed by induction on $k$, and we use the analog of (17): if for some $v \in V(G)$ and $b_{1}, b_{2} \in B(G), \pi\left(b_{i}\right)=\left(v, v_{i}\right)$, and $\left(b_{1}, b_{2}\right) \neq\left(b_{i_{p}}, b_{j_{p}}\right)$ for all $p=1, \ldots, n$, then

$$
\begin{aligned}
R(G ; & \left.\left.; b_{i_{1}}, b_{j_{1}}\right\}, \ldots,\left\{b_{i_{n}}, b_{j_{n}}\right\}\right) \\
= & R\left(G ;\left[b_{1}, b_{2}\right],\left\{b_{i_{1}}, b_{j_{1}}\right\}, \ldots,\left\{b_{i_{n}}, b_{j_{n}}\right\}\right) \\
& +R\left(G ;\left\{b_{1}, b_{2}\right\},\left\{b_{i_{1}}, b_{j_{1}}\right\}, \ldots,\left\{b_{i_{n}}, b_{j_{n}}\right\}\right) .
\end{aligned}
$$

Here the first term corresponds to the partitions, which in addition to above restrictions have bonds $b_{1}, b_{2}$ settled in the same subgraph, while the second one corresponds to the remaining ones. Note that the first term can be treated by the same surgery as that of (17), see Fig. 1. The only additional point is that after accomplishing this transformation one has to revise the list of pairs of bonds $\left\{b_{i_{1}}, b_{j_{1}}\right\}, \ldots,\left\{b_{i_{n}}, b_{j_{n}}\right\}$, because it may happen that for some index, say, $i_{1}, b_{i_{1}}=b_{1}$ or $b_{2}$, and so one has to change $b_{i_{1}}$ to $b_{12}$. Note also, that while each pair of bonds $\left(b_{i_{p}}, b_{j_{p}}\right)$ from our list shares a common site when it enters the list at some step, it can be separated later in the result of further surgeries. This, however, is irrelevant and is mentioned here only in order to clarify the picture.

The proof now goes by successive application of the formula (19). While the first term of the right-hand-side of (19) can then be claimed to have the necessary sign by the applying surgery of Fig. 1 and then the induction hypothesis, the 


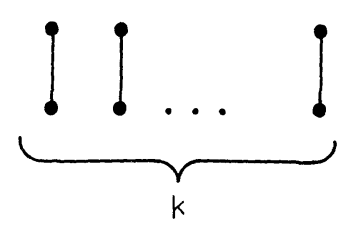

Fig. 2. $G_{k}$ : the simplest graph $G$ with $\#(\partial G)=2 k$

second one cannot be gotten rid of with the help of induction. However, the list of separated pairs of bonds grows longer after each application of (19). The process terminates when each pair of adjacent bonds of $G$ will enter it. Let us denote this final term by the *-superscript.

There are now two possibilities. First is that for some $v \in V(G), \# S(v)>1$. But in that case we claim that

$$
R^{*}\left(G ;\left\{b_{i_{1}}, b_{j_{1}}\right\}, \ldots\right)=0 ;
$$

indeed, there are no allowed partitions in that case! This is because after the last step each bond $b \in S(v)$ has to be in different subgraph, say, $\Gamma(b)$, and so $v \in \partial \Gamma(b)$ for all such $b-s$, which is not allowed by our definition.

So we are left with the remaining case when $\# S(v)=1$ for all $v \in V(G)$. That means that the $G$ itself consist of $k$ disjoint bonds, $b_{1}, \ldots, b_{k}$, see Fig. 2. So we are left to check (18) only for this graph, which is also the initial step of the induction. We shall denote this graph by $G_{k}$.

Lemma 1. Let $G$ be a disjoint union of $G_{1}$ and $G_{2}$, with $\partial G_{1} \neq \emptyset, \partial G_{2} \neq \emptyset$. Then $R(G)=0$.

Proof of the lemma. The above statement can be deduced straightforwardly from the combinatorial definition. However, in the present paper we can enjoy the connection (13) between our $R(G)$ and the Ursell functions. Namely, if one has to evaluate $R(m)$ for some graph (or current) $m=\left\{m_{b}\right\}$ by formula (13), one is allowed to put all interaction constants $J_{b}$ for the bonds $b$ with $m_{b}=0$, to be zero prior to differentiating. But in the case of a disjoint graph $G$ the Ursell function itself is equal to zero, as it can easily be seen from (1), and the lemma follows.

To see that (18) holds for $G=G_{k}$, we again use induction, this time in $k$. For $k=1, R\left(G_{1}\right)=1$ by definition, and in this case the list of divorced pairs of bonds is necessarily empty. Suppose (18) holds for $k-1$ and for all lists of separated pairs. It is easy to see that

$$
\begin{aligned}
& R\left(G_{k} ;\left\{b_{1}^{\prime}, b_{1}^{\prime \prime}\right\}, \ldots,\left\{b_{q}^{\prime}, b_{q}^{\prime \prime}\right\}\right) \\
& \quad+R\left(G_{k} ;\left[b_{1}^{\prime}, b_{1}^{\prime \prime}\right]\right)+R\left(G_{k} ;\left\{b_{1}^{\prime}, b_{1}^{\prime \prime}\right\},\left[b_{2}^{\prime}, b_{2}^{\prime \prime}\right]\right)+\ldots \\
& \quad+R\left(G_{k} ;\left\{b_{1}^{\prime}, b_{1}^{\prime \prime}\right\}, \ldots,\left\{b_{q-1}^{\prime}, b_{q-1}^{\prime \prime}\right\},\left[b_{q}^{\prime}, b_{q}^{\prime \prime}\right]\right)=R\left(G_{k}\right)=0,
\end{aligned}
$$

where the last equality follows from the lemma and the fact that $G_{k}$ is disconnected for $k \geqq 2$.

So it is enough to show that

$$
(-1)^{k+1} R\left(G_{k} ;\left\{b_{1}^{\prime}, b_{1}^{\prime \prime}\right\}, \ldots,\left\{b_{p-1}^{\prime}, b_{p-1}^{\prime \prime}\right\},\left[b_{p}^{\prime}, b_{p}^{\prime \prime}\right]\right) \leqq 0
$$


for all $p$ and all lists of pairs of bonds. But the last expression corresponds to the partitions where $b_{q}^{\prime}$ and $b_{q}^{\prime \prime}$ are forced to be in the same subgraph, hence

$$
\begin{aligned}
& R\left(G_{k} ;\left\{b_{1}^{\prime}, b_{1}^{\prime \prime}\right\}, \ldots,\left\{b_{p-1}^{\prime}, b_{p-1}^{\prime \prime}\right\},\left[b_{p}^{\prime}, b_{p}^{\prime \prime}\right]\right) \\
& =R\left(G_{k-1} ;\left\{\bar{b}_{1}^{\prime}, \bar{b}_{1}^{\prime \prime}\right\}, \ldots,\left\{\bar{b}_{p-1}^{\prime}, \bar{b}_{p-1}^{\prime \prime}\right\}\right),
\end{aligned}
$$

where the bars correspond to the revision of our list due to factorisation of the graph $G_{k}$, which identifies the bonds $b_{p}^{\prime}, b_{p}^{\prime \prime}$. The last expression has the desired sign due to induction hypothesis, and the theorems follow.

\section{Conclusion}

The method of this paper can be used to derive other correlation inequalities. For example, by a slight modification of it, the analogous representation can be obtained for the three-site Ursell function $U_{3}\left(\sigma_{1}, \sigma_{2}, \sigma_{3}\right)$ in the presence of the external magnetic field (otherwise $U_{3}=0$ ), which is manifestly negative for positive fields, thus recovering the GHS inequality.

\section{References}

1. Newman, C.M.: Contribution to "Constructive quantum field theory", Velo, G., Wightman, A.S. (eds.). Lecture Notes in Physics, Vol. 25. Berlin, Heidelberg, New York: Springer 1973

2. Feldman, J.: On the absence of bound states in the $\lambda \varphi_{2}^{4}$ quantum field model without symmetry breaking. Canad. J. Phys. 52, 1583 (1974)

3. Griffiths, R.B.: Correlations in Ising ferromagnets. I. J. Math. Phys. 8, 478 (1967)

4. Griffiths, R.B., Hurst, C.A., Sherman, S.: Concavity of magnetization of an Ising ferromagnet in a positive external field. J. Math. Phys. 11, 790 (1970)

5. Lebowitz, J.: GHS and other inequalities. Commun. Math. Phys. 35, 87 (1974)

6. Cartier, P.: In: Lecture Notes in Mathematics, Vol. 383. Berlin, Heidelberg, New York: Springer 1974, p. 242

7. Percus, J.K.: Correlation inequalities for Ising spin lattices. Commun. Math. Phys. 40, 283 (1975)

8. Sylvester, G.S.: Representations and inequalities for Ising model Ursell functions. Commun. Math. Phys. 42, 209 (1975)

9. Aizenman, M.: Geometric analysis of $\varphi^{4}$ fields and Ising models. Parts I and II. Commun. Math. Phys. 86, 1 (1982)

10. Fröhlich, J.: On the triviality of $\lambda \varphi_{d}^{4}$ theories and the approach to the critical point in $d \geq 4$ dimensions. Nucl. Phys. B 200 FSU, 281 (1982)

Communicated by Ya. G. Sinai

Received June 1, 1985 